\title{
20 ways of removing a nasal foreign body in the emergency department
}

\section{Tian-Tee $\mathrm{Ng}^{*}$}

Department of Surgery, Frankston Hospital. Peninsula Health, PO Box 52, Hastings Road, Frankston 3199, Victoria, Australia

\author{
Abstract \\ Method: We have listed the 20 possible methods one could use to remove NFB in an ED setting. \\ Results \\ 1. Positive Pressure (Child) Method \\ 2. Positive Pressure (Parent) Method - Also known as the kissing technique \\ 3. Modified Positive Pressure (Parent) Method - It is a modification to the kissing technique \\ 4. Positive Pressure with Ambu bag \\ 5. Positive-Pressure Device \\ 6. Beamsley Blaster Positive-Pressure Device \\ 7. Saline Washout Technique \\ 8. $\quad$ Cyanoacrylate (super glue) \\ 9. Catheters \\ 10. The Katz extractor \\ 11. Using a Magnetic Device \\ 12. Instrumentation - Alligator/Crocodile forceps \\ 13. Instrumentation - Tilley nasal packing forceps \\ 14. Instrumentation - Jobson-Horne Probe / ring curette / wax loop \\ 15. Instrumentation - Right angle probe \\ 16. Instrumentation - Frazier suction catheter \\ 17. Instrumentation - Cut-down flexible suction catheter \\ 18. Instrumentation-Refashioned / bent paper clips \\ 19. Snare Technique - Wire loop snare grasped by hemostat \\ 20. "Hook-Scope" Technique for Endoscopic Extraction of NFB
}

Background: Nasal foreign body (NFB) is a common presentation to the emergency department (ED). It is often a dilemma for the treating physician to choose the best method as removal of NFB can be challenging, frustrating and distressing for the physician, the patient, and the patient's parents.

Discussion: Any of the chosen methods has its own benefits and most are suitable for some but not all NFB shapes. In addition, for the removal of NFB with any technique to be successful, four prerequisite needs to be fulfilled which are: a well restrained patient, a good head light with optimal illumination, a nasal speculum and decongested nasal cavity.

Conclusion: The child usually allows only one or two attempts at most for removal of NFB, hence assess the child carefully and if you do not feel you are the right person for the job, get help from seniors or direct referral to the ENT team should you anticipate a difficult removal of a NFB.

\section{Objective}

Nasal foreign body (NFB) is a not an uncommon presentation to the emergency department (ED) and makes up approximately $0.1 \%$ of paediatric emergency department visits [1]. Most commonly found NFB are object of interest to children such as beads, toys, jewels, sweets, stones, batteries and even magnets. For the dilemma facing the
Correspondence to: Dr. Tian-Tee Ng, ENT Unit, Department of Surgery, Frankston Hospital, Frankston 3199, Victoria, Australia, Tel: +61-0426 266 890; E-mail: tntdynamites@yahoo.com

Key words: foreign body, emergency department, nasal, removal

Received: April 05, 2016; Accepted: April 22, 2016; Published: April 26, 2016 
treating physician is to choose the best method of removing the NFB. Removal of NFBs can be challenging, frustrating and distressing for the physician, the patient, and the patient's parents. In this article, we report 20 ways of removing NFBs in the emergency department.

\section{Methods}

A literature search was performed to look for additional ways of removing NFB other than those we routinely apply. Thus by examining published methods, coupled with our experiences, we have compiled a list of all the possible methods one could employ in removing NFB in an ED setting.

\section{Results}

\section{Positive Pressure (Child) Method [1]}

Technique: Child to exhale forcibly through the nostril containing the object with the opposite nostril occluded and the mouth closed.

Good for: Solid object e.g. beads

Note: Difficult or impossible for young patients to accomplish on their own.

\section{Positive Pressure (Parent) Method [1,2] - Also known as the kissing technique}

Technique: Parent uses his or her mouth to apply positive pressure into the patient's mouth with simultaneous occlusion of the contralateral nostril [1].

Good for: Solid object e.g. beads

Note: Potentially less emotionally traumatic for the child than direct physical removal of the object ${ }^{1}$ and good for very young patients.

\section{Modified Positive Pressure (Parent) Method - a modification of the kissing technique}

Technique: Using drinking straw, or similar tubing, between the parent's mouth and child's mouth, the child is instructed to make a tight seal, as if drinking, and the parent delivers a quick puff $[1,3]$.

Good for: Solid object e.g. beads

Note: Risk of bleeding from tip of straw or rigid tubing if patient moves.

\section{Positive Pressure with Ambu bag}

Technique: Oral insufflation with an ordinary Ambu bag [1].

Good for: Solid object e.g. beads

Note: Reasonable alternative in instances when parent and child have difficulty cooperating with the parent-applied mouth-to-mouth positive pressure.

\section{Positive-Pressure Device [4]}

Technique: Nasal occlusion device consisting of a medium or large disposable headset attached to an $8 \mathrm{~F}$ feeding tube (Figure 1). The headset includes a hose that is connected to a standard oxygen outlet, with an oxygen flow rate of $15 \mathrm{~L} / \mathrm{min}$ which is equivalent to an output pressure of 100 to $160 \mathrm{~mm} \mathrm{Hg}$. With the patient restrained and properly positioned (sitting on parent's lap), the occlusion device is connected to the oxygen hose. The oxygen outlet is opened with a pressure of $15 \mathrm{~L} /$ min, and the hose bent to occlude passage of pressure. After the device

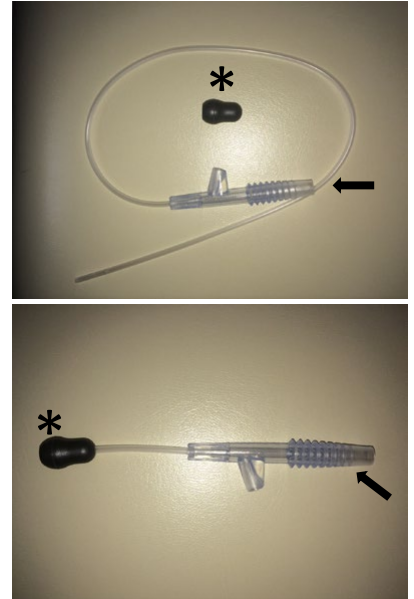

Figure 1. An $8 \mathrm{~F}$ feeding tube (arrow); disposable headset (asterisk) ${ }^{4}$.

was placed in the unaffected nostril, the pressure was suddenly released and the device was immediately removed.

Good for: Solid object e.g. beads

Note: This device is comfortable, easy to use, and regulates sufficient positive pressure necessary to expel a NFB.

\section{Beamsley Blaster Positive-Pressure Device [4]}

Technique: Uses an oxygen tube adapter that provides unmodulated pressure in the posterior nasopharynx to eject the NFB.

Good for: Solid object e.g. beads

Note: Barotrauma manifested as periorbital subcutaneous emphysema has been reported as a complication and is therefore a risk.

\section{Saline Washout Technique [1,5]}

Technique: A bulb syringe filled with approximately $7 \mathrm{ml}$ of sterile normal saline is placed in the opposite nostril. The bulb syringe is advanced several centimetres into the nasal cavity so that a tight seal is maintained. The bulb syringe is forcibly squeezed and the object is propelled out by the flow of saline back through the nasal passage that contains the foreign body.

Good for: Friable foreign body

Note: Potential reflux of saline and/or nasal contents into the eustachian tubes and potential aspiration of the foreign body [5]. However, no known adverse event has been reported by investigators who have regularly performed this technique to obtain nasal specimens for research studies [5].

\section{Cyanoacrylate (super glue)}

Technique: Cyanoacrylate applied to the end of a plastic swab stick for the removal of nasal foreign bodies ${ }^{1}$. The stick must be pressed and held onto the NFB for 60 seconds before being withdrawn.

Good for: Solid object e.g. beads

Note: Any cyanoacrylate stuck and remaining on the skin can be removed using $3 \%$ hydrogen peroxide or acetone.

\section{Catheters}

Technique: Any catheters such as Foley catheters, Fogarty vascular catheters, or Fogarty biliary balloons can be used $[1,6]$. After checking 
the integrity of the balloon, the catheter is inserted above and distal to the foreign object (passing the catheter below the object can potentially drive the object into a tighter position). Once beyond the foreign body, the balloon is inflated with a predetermined amount of saline ( $1 \mathrm{~mL}$ for a no. 4 Fogarty catheter, $2-3 \mathrm{~mL}$ for a no. 6 Fogarty or $8 \mathrm{~F}$ Foley catheter) and maintained at that size with pressure from the practitioner's thumb. Gentle traction is then applied to remove the object [1] (Figure 2).

Good for: Good for NFB that cannot be visualised, but has a reliable history or signs of NFB lodgement (unilateral nasal obstruction or discharge) [6].

Note: Dwyer has reported more than 200 successful NFB removals in children via this technique which has become his sole strategy in removing NFB [6].

\section{The Katz extractor [1]}

Technique: The success of the catheter method has led to the development of a disposable catheter made specifically for the removal of NFB called the Katz extractor [1]. The Katz extractor catheter is smaller than the catheters mentioned above, which results in a greater chance of the catheter being passed beyond the foreign body $\mathrm{S}$

\section{Good for: Good for NFB that cannot be visualised}

Note: Easy to use. It only takes three simple steps to complete an extraction with the Katz Extractor: - Insert / Inflate / Extract.

\section{Using a Magnetic Device [7]}

Techniques: A strong magnet is used; for example in the ED setting it could be the magnet used for deactivating pacemakers.

Good for: Metallic NFB and button batteries

Notes: If the NFB is a button battery this is a medical emergency; and must be removed as soon as possible.

\section{Instrumentation - Alligator/Crocodile forceps [8] (Figure 4)}

Technique: Using one hand to elevate the nasal tip of the patient, the other hand inserts forceps into the nasal cavity to grasp the NFB

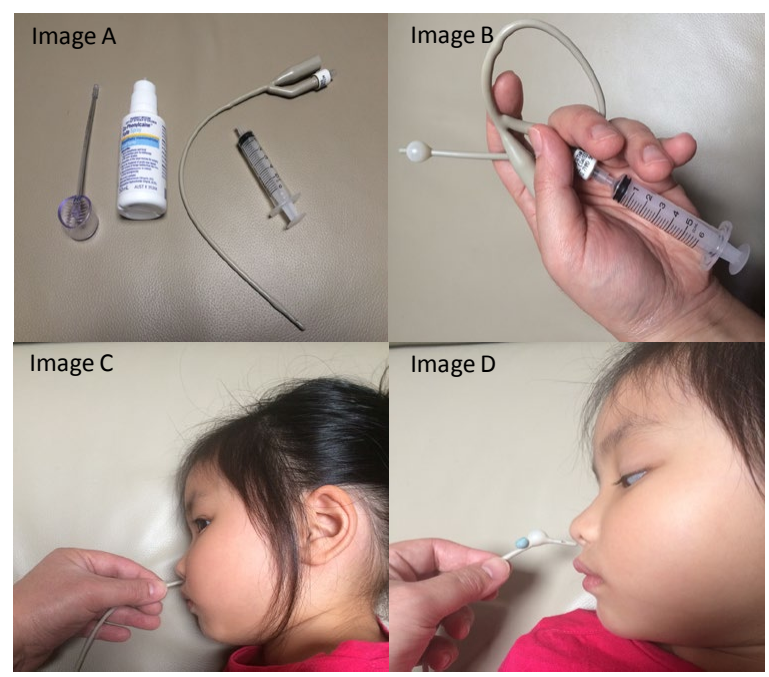

Figure 2. (Image A)Equipment required. (Image B) Determining required balloon size specific to each child. (Image C)Balloon inflated once predetermined insertion length achieved. (Image D) Gentle, steady traction allows FB removal ${ }^{6}$.
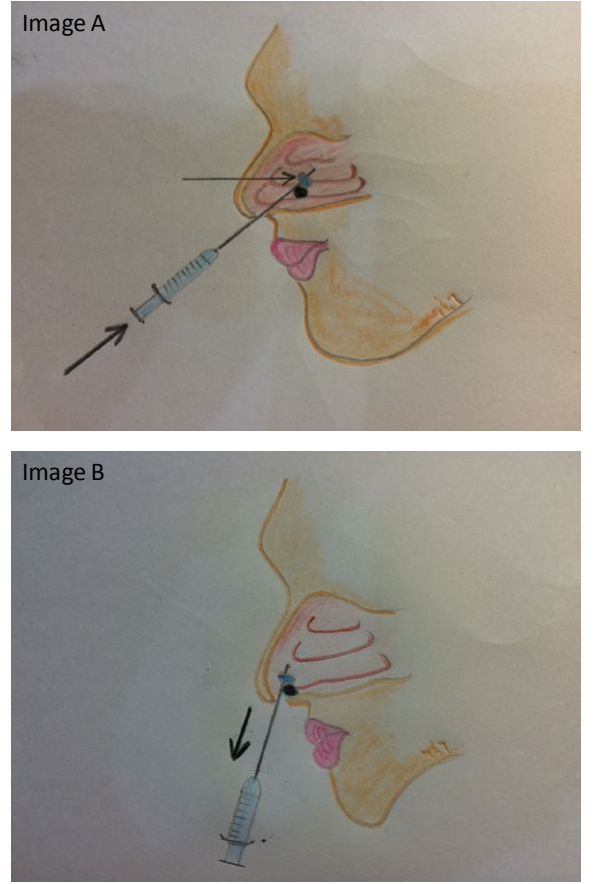

Figure 3. (Image A) Katz extractor balloon inflated with air from connected syringe (Image B) Katz extractor removed with nasal foreign body ${ }^{1}$.

Good for: Any firm to hard NFB

Note: Not suitable for round NFB as repeated attempts to grasp only for object to escape could propel the NFB further back into the nasal cavity.

\section{Instrumentation - Tilley nasal packing forceps [8] (Figure 4)}

Technique: For removal of NFB deemed too large to be grasp with Alligator forceps

\section{Good for: Any firm to hard NFB}

Note: Not suitable for round NFBs as forceps are likely to slip off and propel the NFB back further into the nasal cavity.

\section{Instrumentation - Jobson-Horne Probe/ring curette/wax loop [8] (Figure 4)}

Technique: With nasal tip of the patient elevated using the other hand, the probe is inserted into the nasal cavity beyond the NFB, and then angle the tip is angled to allow the probe to propel the NFB out ahead of it as the probe is withdrawn from the nose ${ }^{8}$.

Good for: Solid round object e.g. beads

Note: This author has found this method to have a $100 \%$ success rate and have consequently adopted this method as my practice of choice in removing NFB.

\section{Instrumentation - Right angle probe [9] (Figure 4)}

Technique: The probe is manoeuvred alongside and past the NFB, then rotated it so that the right-angle is behind the NFB, and then withdrawn along with the object [9].

Good for: Solid object e.g. stones, Lego ${ }^{\circledR}$ pieces

Note: Risk of epistaxis if patient moves or struggles while probe is in the nose 


\section{Instrumentation - Frazier suction catheter [9] (Figure 4)}

Technique: Place the end of the suction catheter on the surface of the object, apply suction and gently pull out the catheter with the NFB attached.

\section{Good for: Solid round object e.g. beads}

Note: Risk of epistaxis if patient moves or struggles while catheter is in the nose.

\section{Instrumentation - Cut-down flexible suction catheter [9] (Figure 4)}

Technique: Place the end of the suction catheter on the surface of the object, apply suction and gently pull out the catheter with the NFB attached.

\section{Good for: Solid round object e.g. beads}

Note: Less risk of epistaxis compared to using a Frazier suction catheter

\section{Instrumentation- Refashioned / bent paper clips (Figure 5)} loop.

Technique: Works like a Jobson-Horne Probe / ring curette / wax

Good for: Solid objects e.g. beads

Note: Use it when Jobson-Horne Probe / ring curette / wax loop is needed but cannot be found.

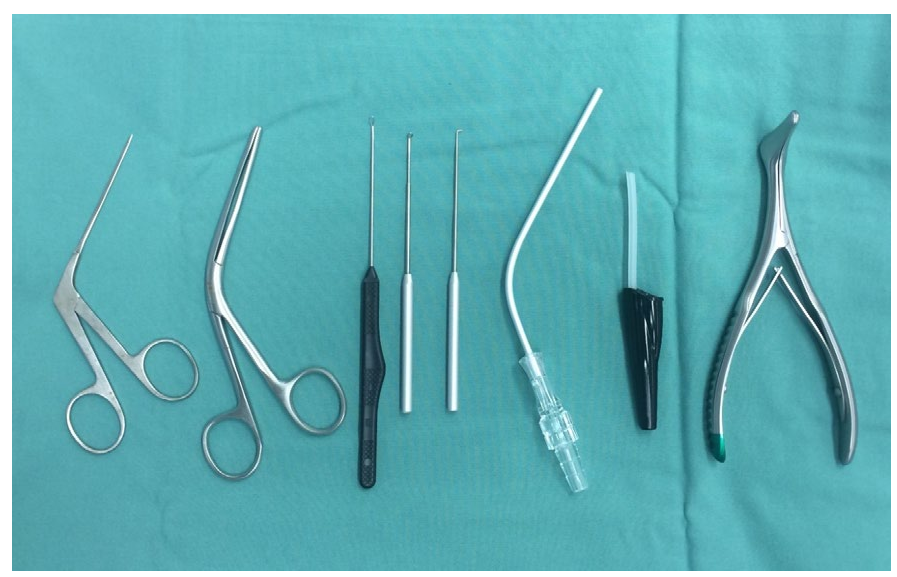

Figure 4. From left to right: Alligator/Crocodile forceps, Tilley nasal packing forceps, wax loop, ring curette, right angle probe, Frazier suction catheter, cut-down flexible suction catheter and Killian nasal speculum.

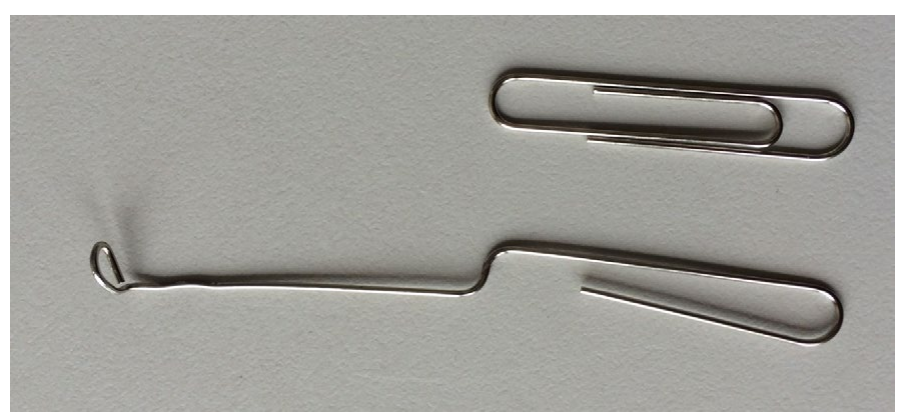

Figure 5. Refashioned / bent paper clips

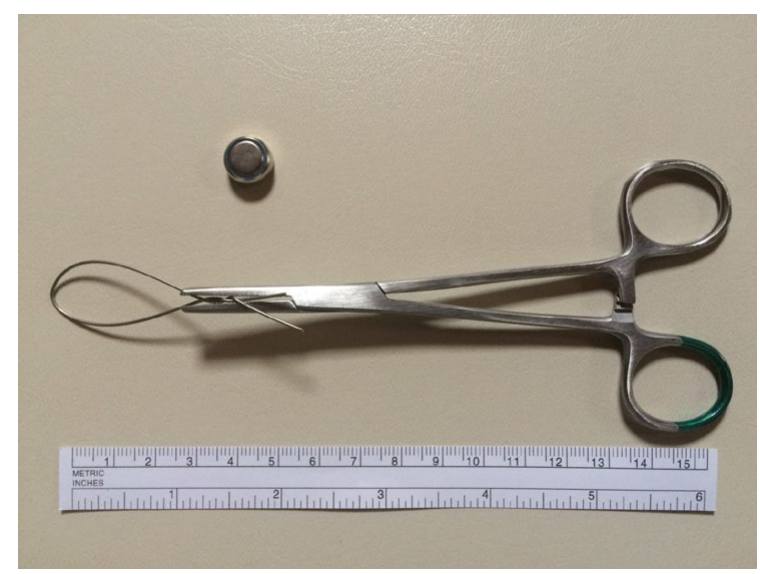

Figure 6. Example of wire loop snare grasped by haemostat after successful atraumatic removal of nasal foreign body (battery) ${ }^{10}$.

\section{Snare Technique - Wire loop snare grasped by haemostat}

Technique: A 24-gauge wire "snare" loop is created, then held and its position guided by a haemostat. The snare is inserted into the nasal aperture, and used to separate a plane between the NFB and the septal, turbinate and nasal floor mucosa until all sides were free. Once the posterior free edge of the NFB is palpable with the loop, it is rotated 90 degrees and retracted outward, freeing the NFB and bringing it forward (Figure 6).

Good for: Impacted NFB e.g.: button battery

Note: This technique is noted to be a rapid, atraumatic, and effective means for the removal of difficult NFBs [10].

\section{"Hook-Scope" Technique for Endoscopic Extraction of NFB}

Technique: A flexible nasal endoscope ( $3.7 \mathrm{~mm}$ diameter) connected to a video system is needed. First assess the NFB and surrounding nasal cavity using the nasal endoscopy. Upon location of NFB, the scope's head is then turned superiorly to identify the nasal area above the superior margin of the NFB, which will be the pathway for the scope to travel through. The existence of such a pathway is a prerequisite for the success of this technique. The endoscope is subsequently advanced above and posterior to the NFB, and, finally descends to the area facing the nasal floor in a manoeuvre that bypasses the object (Figure 7). After a quick assessment of the posterior extension of the NFB and the status of the posterior nasal cavity, turned anteriorly towards the object, encasing the NFB like a hook. The NFB is then disengaged and mobilized, by gently pulling the scope anteriorly outward towards the nasal vestibule, keeping the NFB locked in the flexed mode and the object enclosed within its hook. Following successful extraction, a diagnostic nasal endoscopy is performed to reassess the entire nasal cavity.

The core of this technique is that the NFB is actually embraced by the endoscope, which subsequently acts as an extractor [11].

Good for: Posteriorly located and round objects that are difficult to grasp [11].

Note: Particular attention must be paid in order not to inadvertently dislocate the object towards the choana.

The endoscope may become damaged in the hands of unfamiliar operator. 


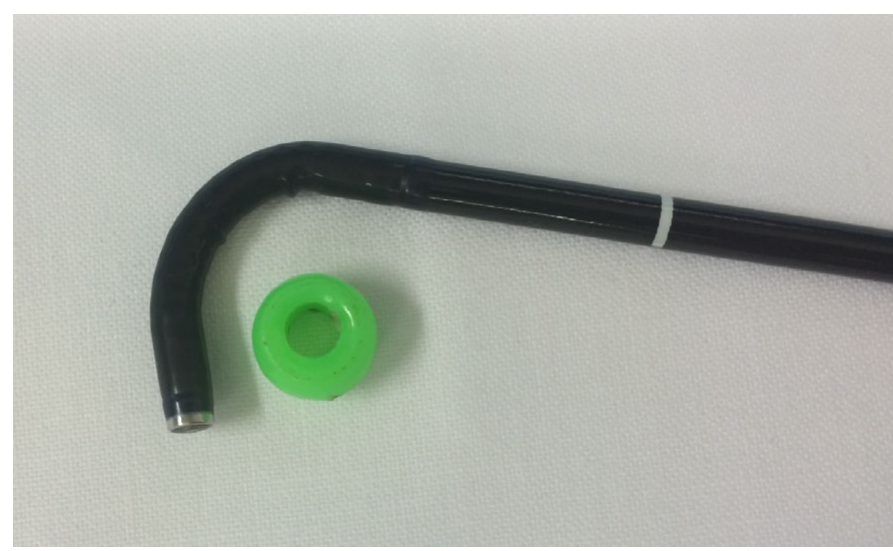

Figure 7. Two-dimensional reproduction of the hook-scope manoeuvre ${ }^{11}$.

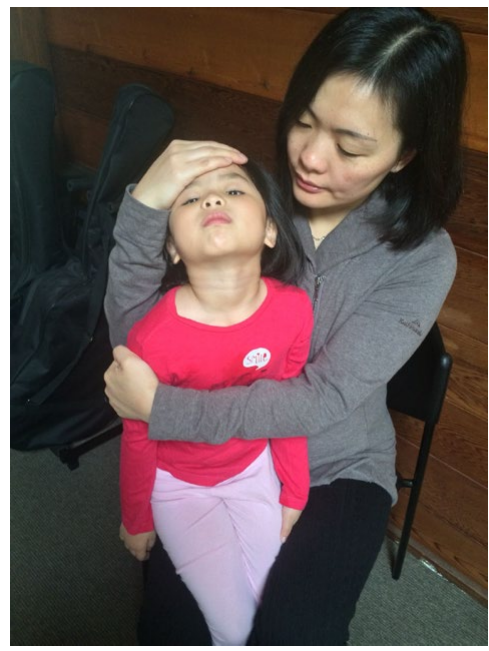

Figure 8. The correct method to restrain a child.

\section{Discussion}

Any of the chosen method, from a non-instrumental method such as the positive pressure to instrumental extraction, has its own benefits and advantages. For any removal of NFB to be successful, there are four prerequisite that needs to be fulfilled; these are:

First - a restrained patient. A moving or mobile patient is at risk of epistaxis with instrumentation which will make the process of removing the NFB even more difficult, visualisation of the NFB almost impossible and risk posterior dislodgement of the NFB. A child can be safely and securely restrained by having the child sit on their parent's lap with the body facing to the front. The parent has one arm over the child's body and arms, and the parent's other arm holds onto the child's forehead, pushing the back of the child' head against the parent's chest. The child's lower limbs are securely locked between the parent's thighs (Figure 8).

Second - a good head light, eg Vorotek headlight. I cannot over stress the importance of having a good otolaryngology headlight as it frees both the operator's hands and gives a focus illumination into the tiny nasal cavities of the child.

Third - a nasal speculum, e.g. Killian nasal speculum (Figure 4). In my early years as a junior registrar I missed NFB in two paediatric patients when examining without nasal speculum. Fortunately, both patients presented back for review and had proper nasal examination with nasal speculum where the NFB was identified and removed safely.

Fourth - nasal decongestion and analgesia with local topical agent (cophenylcaine nasal spray). This is important as it decongests and numbs the nasal cavity to aid in the removal of NFB. The topical spray is dose dependent, hence be careful when using it on paediatric patients.

\section{Conclusions}

It is down to the operator to find the method he or she is most comfortable with to remove NFBs. Bear in mind, the child usually allows only one or two attempts at most for removal of NFB, hence assess the child carefully and if you do not feel like you are the right person for the job, get help from seniors or direct referral to the ENT team if the removal of NFB is anticipated to be difficult. If all else fails, no further attempt in removing the NFB in ED should be made, and NFB removal under general anaesthesia would be the next course of action.

\section{Acknowledgments}

I would like to thank Dr. Vicky Tobin, Dr. David Hunter-Smith Ms. Ebony Dejager and Frankston Hospital Library.

\section{Financial support}

This project was not financially supported from any external sources and was fully funded by the Department of Surgery, Frankston Hospital.

\section{Conflict of interest}

The authors declare no conflict of interest.

\section{References}

1. Kiger JR, Brenkert TE, Losek JD (2008) Nasal foreign body removal in children Pediatric Emergency Care 24: 785-792. [Crossref]

2. Colleen T, Acheson J, Coats TJ (2010) Nasal foreign bodies in children: kissing it better. Emergency Medicine Journal 27: 712-713. [Crossref]

3. Benjamin E, Harcourt J (2007) The modified 'Parent's Kiss' for the removal of paediatric nasal foreign bodies. Clinical Otolaryngology 32: 120-121. [Crossref]

4. de la O-Cavazos M, Ríos-Solís J, Montes-Tapia F, Elizondo-Omaña R, Cantu-Moreno D, (2014) Pediatric Emergency Care 30: 94-96. [Crossref]

5. Lichenstein R, Giudice EL (2000) Nasal wash technique for nasal foreign body removal. Pediatric Emergency Care 16: 59-60. [Crossref]

6. Dwyer D (2015) Foley catheter technique for nasal foreign body removal in children Emergency Medicine Australasia 27: 495-496.

7. Alletag MJ, Jacobson D, Santucci K, Riera A (2014) Nasal disc battery removal novel technique using a magnetic device. Pediatric Emergency Care 30: 488-490. [Crossref]

8. Motbey J (2001) How to remove foreign body from a child's nose. Medicine Today 2: $106-107$.

9. Craig SS, Cheek JA, Seith RW, West A (2015) Removal of ENT foreign bodies in children. Emergency Medicine Australasia 27: 145-147.

10. Fundakowski CE, Moon S, Torres L (2013) The snare technique: a novel atraumatic method for the removal of difficult nasal foreign bodies. Journal of Emergency Medicine 44: 104-106. [Crossref]

11. Giourgos G, Matti E, Colombo A, Pagella F (2009) "Hook-scope" technique for endoscopic extraction of nasal foreign bodies. Laryngoscope 119: 1203-1205. [Crossref]

Copyright: (C2016 Tian-Tee Ng. This is an open-access article distributed under the terms of the Creative Commons Attribution License, which permits unrestricted use, distribution, and reproduction in any medium, provided the original author and source are credited. 